tongue movements with the initiation of $\mathrm{CBZ}$ treatment for complex partial seizures, and the absence of other etiologies, suggests that CBZ was the likely cause. Additional cases of carbamazepine-related tics and other movement disorders have been reported.

The contingent negative variation (CNV), a slow brain potential which may reflect the level of central dopamine and is a measure of arousal and attention, has been studied in patients with Tourette syndrome (Weate SJ, Drake ME Jr et al. Clin EEG Oct 1993;24:188-191). Patients had higher CNV amplitude and more frequent postimperative negative variation than controls, possibly explained by dopaminergic excess. Medication was not a factor.

\title{
BENIGN HEREDITARY CHOREA
}

The clinical manifestations, differential diagnosis, and treatment of benign hereditary chorea (BHC) are reviewed from the Indiana University and University of Minnesota Medical Schools. The diagnosis of BHC is by exclusion. The most common age of onset is about 1 year and walking is often delayed because of clumsiness and falling. After a short period of progression in severity, movements reach a plateau and may sometimes appear to improve. IQ is normal or low-average. In most reported families, the inheritance is autosomal dominant. Treatment with various drugs, eg. haloperidol, chlorpromazine, and prednisone, is only partially effective, and the chorea is usually refractory. (Wheeler PG, Weaver DD, Dobyns WB. Benign hereditary chorea. Pediatr Neurol Sept/Oct 1993; $2: 337-340$ ). (Respond: Dr Wheeler, Dept of Medical and Molecular Genetics, IB 130, Indiana University Medical Center, 975 West Walnut St, Indianapolis, IN 46202).

COMMENT. BHC is an uncommon form of childhood chorea that has been mistaken for Huntington disease. The recognition of this benign, nonprogressive form of chorea of early onset and without dementia will avoid unnecessary prolonged hospitalization and the risk of invasive diagnostic procedures.

\section{BILATERAL STRIATAL SYNDROMES}

The clinical manifestations and outcome in 13 patients with bilateral basal ganglia lesions and neurological dysfunction are reported from the Child Neurology Unit, Vall D'Hebron University Hospital, Barcelona, Spain. Lesions were demonstrated by CT, MRI, or ultrasound, and in 4 patients who died the pathology was subacute necrotizing encephalomyelopathy (SNE) (2), hypoxic -ischemic encephalopathy (HIE) (1), and intracranial hemorrhage (1). Extrapyramidal signs included dystonia in 9 patients, hypotonia in 2, athetosis 1 , and rigidity 1 . Consciousness was altered in 5 , and seizures occurred in 3 . 
The outcome was poor in the majority, with motor sequelae and/or cognitive impairment. (Roig M et al. Bilateral striatal lesions in childhood. Pediatr Neurol Sept/Oct 1993;9:349-358). (Respond: Dr Roig, Child Neurology Unit, Hospital Materno-Infantil Vall D'Hebron, Paseo del Vall D'Hebron s/n, Barcelona, Spain).

COMMENT. Based on a review of the literature, the authors tabulate the causes of infantile bilateral striatal syndrome (IBSS) under acute and subacute/chronic categories. Acute causes include SNE, HIE, trauma, hemolytic-uremic syndrome, infections, and MELAS. Chronic causes include SNE, Huntington disease, Wilson disease, acanthocytosis, SSPE, glutaric aciduria, and familial metabolic disorders. The pathological findings were necrosis and neuronal loss. CT demonstrated hypodensities and the MRI showed hyperintense T2 weighted images in the basal ganglia.

The physiology of basal ganglia disorders is reviewed and an hypothesis for basal ganglia function is proposed by Hallett M, Clinical Director, NINDS, NIH, Bethesda (Can I Neurol Sci Aug 1993;20:177-183). A direct path linking the putamen and globus pallidus is a positive feedback circuit that selects specific motor synergies to carry out a desired action whereas an indirect path inhibits these synergies, eg. dystonia results from overactivity of the putamen and the direct pathway; chorea is explained by underactivity of the indirect pathway.

\section{HEADACHE DISORDERS}

\section{MIGRAINE DIAGNOSES AND SYMPTOM PATTERNS}

The diagnostic rates based on various criteria and symptom complexes of children and adolescents with chronic headache were evaluated in 88 consecutive patients who attended the Pediatric Headache Clinic at the University of Maryland School of Medicine, Baltimore, 1989-1991. The male/female ratio was 1 (43:45). The mean age 12.5 years; 44 Caucasian and 40 African-American. Clinical migraine was diagnosed in $63(74 \%)$, of which only $14 \%$ were classic in type. Migraine versus non-migraine diagnoses were not related to age, gender, or race. When International, Prensky, Vahlquist, and the author's (Gladstein) criteria for migraine were used, the diagnosis was applied in only $50 \%$. Prevalence of migraine diagnosis by the International criteria differed from the other classifications as a function of race, favoring Caucasians over African-American, $61 \%$ to $35 \%$. Minority children were less likely to present with nausea, vomiting, lateralized pain, or food as a precipitant of headache. Children older than 12 years complained more often of radiating pain, scalp tenderness, and abdominal pain. Adolescent males reported higher rates of vomiting and decreased appetite than in females, while adolescent females found stress a more frequent precipitant. Older children and adolescents, especially females, used aspirin more frequently than those under 12 years of age. (Gladstein J et al. Diagnoses and symptom 